\title{
Identification of a Calcitriol-Regulated Sp-1 Site in the Promoter of Human CD14 using a Combined Western Blotting Electrophoresis Mobility Shift Assay (WEMSA)
}

\author{
Alireza Moeenrezakhanlou ${ }^{1,2}$, Devki Nandan ${ }^{1}$ and Neil E. Reiner ${ }^{1 \#}$ \\ ${ }^{1}$ Department of Microbiology and Immunology, University of British Columbia, Faculties of Medicine and Science, and Vancouver Coastal Health Research \\ Institute (VCHRI), Vancouver, British Columbia, Canada, V5Z $3 J 5$. \\ ${ }^{2}$ School of Medicine, Hamadan University of Medical Sciences, Hamadan, Iran. \\ "Corresponding Author: Neil E. Reiner, Division of Infectious Diseases, University of British Columbia, Rm 452D, 2733 Heather Street, Vancouver, BC. \\ Canada, V5Z 3J5. Phone: 604-875-4011; Fax: 604-875-4013; E-mail: ethan@interchange.ubc.ca
}

Submitted: March 23, 2007; Revised: December 10, 2007; Accepted: December 12, 2007

Indexing terms: Electrophoretic Mobility Shift Assay; Transcription Factors; Antigens, CD14

Abbreviations: TF, transcription factor; VDRE, vitamin D response element; WEMSA, western blot electrophoretic mobility shift assay

\begin{abstract}
Calcitriol $\left(1 \alpha, 25\right.$-dihydroxyvitamin $\left.\mathrm{D}_{3}\right)$ induces the expression of $C D 14$ in mononuclear phagocytes. The mechanisms accounting for this have been unclear since the promoter of $C D 14$ does not contain a canonical vitamin D response element (VDRE). Calcitriol has been shown to regulate the activity of the transcription factor Sp-1 and our analysis of the proximal promoter of $C D 14$ indicated the presence of four Sp-1-like binding sequences. To identify which of these sites might be involved in the response to calcitriol, we used a system incorporating an electrophoretic mobility shift assay (EMSA) coupled to Western blot analysis (WEMSA). Using WEMSA, we found that only one of the Sp-1-like binding sequences, located at position -91 to -79 (relative to the transcription start site), bound the transcription factor Sp1. Sp-1 binding to this site was demonstrable using nuclear extracts from control cells. Notably, binding activity was attenuated in nuclear extracts prepared from cells that had been incubated with calcitriol, thus suggesting Sp-1 involvement in calcitriol induction of CD14 expression. Notably, these results show that like EMSA, WEMSA can be broadly applied to aid in the identification of transcription factors involved in regulating gene expression. WEMSA, however, offers a number of distinct advantages when compared with conventional EMSA. Antibodies used for WEMSA often provide less ambiguous signals than those used in EMSA, and these do not have to recognize epitopes under native conditions. In addition, WEMSA does not require the use of labeled oligos, thus eliminating a significant expense associated with EMSA.
\end{abstract}

\section{INTRODUCTION}

The regulation of gene expression in mammalian cells is controlled by approximately 200 transcription factors that interact with distinct DNA binding sites (1). Transcription factors (TF) are proteins involved in the negative and positive regulation of gene expression. Their mechanisms of action may involve either direct binding to specific DNA sequences in promoter regions of target genes, or indirect effects on gene expression through interactions with other proteins directly bound to DNA elements. Each TF is defined by the short DNA sequences that reflects its DNA binding motif (2). TFs usually consist of two functional domains, a DNA binding domain and a regulatory domain that interacts with components of the transcription initiation complex and regulatory proteins, thereby affecting the efficiency of DNA binding and transcription. Typically, gene promoters contain several functional TF-DNA binding sites and in most cases, there is more than one specific DNA binding site for a particular 
TF (3). In addition, promoters of human genes contain many transcription factor binding elements that have a varied range of identities when compared to canonical transcription factor binding elements (4).

Calcitriol induces monocyte differentiation and this is reflected in increased surface expression of both $C D 14$ and $C D 11 b$ (5). Since no canonical vitamin D response element (VDRE) has been identified in the human CD14 promoter, the mechanism of $C D 14$ activation in response to calcitriol is unclear. Recently, the transcription factor CREB has been shown to be activated by calcitriol and to play a role in the regulation of $C D 14$ expression (6). It has also been reported that calcitriol modulates Sp-1 activity and this too may be involved in regulating CD14 expression (7). Sp-1 belongs to a family of transcription factors that bind to GC-rich sequences and is involved in regulating cell growth, apoptosis and angiogenesis (reviewed in 8). Our analysis of the proximal promoter of human CD14 identified four candidate Sp-1-like sequences. This raised the question of which, if any, of these sequences is involved in regulating the $C D 14$ response to calcitriol.

Conventionally, electrophoretic mobility shift assays (EMSA) combined with antibody supershifts are used to investigate the interactions of TFs with specific DNA elements (9). EMSAs are carried out using short synthetic labeled DNA probes corresponding to TF binding sequences in the promoter region of the gene of interest. These probes are then incubated with crude nuclear extracts followed by the electrophoretic separation of the resulting protein-DNA mixture on a polyacryamide or agarose gel in a low ionic strength buffer which results in a mobility shift. In general, an antibody- mediated supershift is then used to confirm the identity of the particular TF involved. In most situations, canonical DNA probes are used for EMSA analysis. However, variability in the DNA binding sequences recognized by TF binding domains may complicate the results. While supershifts can be highly informative, they have limitations. In many supershift assays for example, multiple bands are present which may be diffuse and difficult to interpret. In addition, antibodies capable of mediating a supershift for a particular transcription factor of interest may not always be available because of the requirement that they recognize epitopes under native conditions (10). The very nature of the supershift assay itself also does not allow the re-use of antibodies, thus making this technique somewhat costly. Moreover, EMSA-based supershift assays are carried out in a manner such that they do not permit examination of multiple TFs simultaneously. In a previous report, we used a modification of EMSA to incorporate Western blotting in a combined approach which we referred to as WEMSA (6). The present study again utilizes WEMSA to examine the regulation of Sp-1 binding to the promoter region of CD14 in myeloid cells in response to calcitriol. The findings reported illustrate the general applicability of WEMSA to identify active sequences in gene promoters and their cognate DNA binding proteins. Because Western blotting is incorporated in this assay, the recycling of antibodies is possible in most situations and this significantly reduces the cost normally associated with classical supershift assays. Moreover, suitable antibodies for WEMSA are more generally available since there is no requirement that they must recognize epitopes under native conditions. Importantly, WEMSA also has the advantage of not requiring the use of labeled oligos needed for EMSA. This along with the advantages outlined above, makes this technique an attractive alternative to classical EMSA to study the roles of transcription factors in regulating gene expression.

\section{MATERIALS AND METHODS}

\section{Reagents and Chemicals}

$1 \alpha, 25$-dihydroxyvitamin $\mathrm{D}_{3}$ (calcitriol), was obtained from Calbiochem Corporation (San Diego, CA). RPMI 1640, Hank's Balanced Salt Solution (HBSS), penicillin/streptomycin, protease inhibitor cocktail, PMSF, and poly ( $\mathrm{dI}-\mathrm{dC})$ were purchased from Sigma Chemical Co. (St. Louis, MO). Nitrocellulose membranes were obtained from Bio-Rad Laboratories (Hercules, CA). Unlabelled and labelled Sp-1-like oligos for WEMSA and EMSA were from Invitrogen (Carlsbad, CA). Unlabelled and labelled canonical Sp-1 oligos were purchased from Panomics. Anti-Sp-1 antibody was from Upstate Cell Signaling Solutions (Lake Placid, NY).

\section{Cell Culture}

The human promonocytic cell line THP-1 (acquired from the American Type Culture Collection, Rockville, MD) was cultured at $37^{\circ} \mathrm{C} / 5 \% \mathrm{CO}_{2}$ in RPMI 1640 medium supplemented with $10 \%(\mathrm{v} / \mathrm{v})$ heat-inactivated fetal bovine serum (FBS), penicillin (100 units/ml) and streptomycin $(100 \mu \mathrm{g} / \mathrm{ml})$. For most experiments, prior to use, cells were washed in HBSS and incubated for $5 \mathrm{~h}$ in RPMI/0.5\% FBS for serum starvation. 


\section{Preparation of Nuclear and Cytoplasmic Fractions}

Nuclear and cytoplasmic fractions were prepared based on a protocol from the Skirball Institute of Biomolecular Medicine, NYU Medical Center (6) with minor modifications. Briefly, approximately $2 \times 10^{7}$ THP- 1 cells were used per assay and after treatment the cells were washed twice with HBSS and collected by centrifugation. Subsequent steps were performed on ice. The cells were resuspended in $300 \mu \mathrm{l}$ of fractionation buffer $(10 \mathrm{mM}$ HEPES pH 7.9 containing $50 \mathrm{mM} \mathrm{NaCl}, 0.5 \mathrm{M}$ sucrose, 0.1 mM EDTA, 0.5\% Triton- $X$ 100, 1 mM DTT, $10 \mathrm{mM}$ sodium pyrophosphate, $2 \mathrm{mM} \mathrm{NaF}, 17.5 \mathrm{mM} \beta$-glycerophosphate, $1 \mathrm{mM}$ PMSF and $4 \mu \mathrm{g} / \mathrm{ml}$ aprotinin, $2 \mu \mathrm{g} / \mathrm{ml}$ pepstatin A, and $2 \mu \mathrm{g} / \mathrm{ml}$ leupeptin and incubated on ice for $5 \mathrm{~min}$. Nuclei were collected at $4500 \times \mathrm{g}$ for $5 \mathrm{~min}$ at $4^{\circ} \mathrm{C}$. Nuclei were washed and resuspended in $500 \mu \mathrm{l}$ buffer A (10 mM HEPES pH 7.9 containing $10 \mathrm{mM} \mathrm{KCl}, 0.1 \mathrm{mM}$ EDTA, 0.1 $\mathrm{mM}$ EGTA, $1 \mathrm{mM}$ DTT, $1 \mathrm{mM} \mathrm{PMSF}$, and $4 \mu \mathrm{g} / \mathrm{ml}$ aprotinin, $2 \mu \mathrm{g} / \mathrm{ml}$ pepstatin $\mathrm{A}$, and $2 \mu \mathrm{g} / \mathrm{ml}$ leupeptin) and pelleted at $4500 \times \mathrm{g}$ for $5 \mathrm{~min}$ at $4^{\circ} \mathrm{C}$. Nuclear pellets were resuspended in $40 \mu \mathrm{l}$ of buffer B (10 mM HEPES pH 7.9, $500 \mathrm{mM} \mathrm{NaCl}, 0.1 \mathrm{mM}$ EDTA, $0.1 \mathrm{mM}$ EGTA, 0.1\% IGEPAL, $1 \mathrm{mM}$ DTT, $1 \mathrm{mM} \mathrm{PMSF}$, and $4 \mu \mathrm{g} / \mathrm{ml}$ aprotinin, 2 $\mu \mathrm{g} / \mathrm{ml}$ pepstatin $\mathrm{A}$, and $2 \mu \mathrm{g} / \mathrm{ml}$ leupeptin) and mixed by extensive vortexing over 10-15 $\mathrm{min}$ at room temperature. Tubes were kept cold by placing them on ice intermittently during vortexing. Samples were centrifuged at $11,600 \times \mathrm{g}$ for $20 \mathrm{~min}$ at $4^{\circ} \mathrm{C}$ and supernatants representing crude nuclear extracts were then transferred to new tubes and adjusted to a final concentration of $10 \%$ glycerol. The aliquots were stored at $-70^{\circ} \mathrm{C}$.

\section{Preparation of the Oligonucleotides}

The promoter of CD14 was analyzed by TFSEARCH software (http://www.cbrc.jp/research/db/TFSEARCH.html) and four different candidate $\mathrm{Sp}-1$ sequences were identified. Synthesized solutions of sense and antisense oligonucleotides were prepared at $10 \mu \mathrm{M}$ concentration in $20 \mathrm{mM}$ Tris $\mathrm{pH}$ 8.0. Sense and antisense oligos were then annealed by heating at $95^{\circ} \mathrm{C}$ for $5 \mathrm{~min}$ and cooled to room temperature for $15 \mathrm{~min}$ before storage at $-20^{\circ} \mathrm{C}$.

\section{Electrophoretic-Mobility Shift Assay}

2-5 $\mu$ g of nuclear extract was used for EMSA according to the manufacturer's instructions (Panomics, EMSA GelShift Kit). Briefly, nuclear extracts containing equal amounts of protein for each sample were incubated with poly (dI-dC) $(1 \mu \mathrm{g} / \mu \mathrm{l})$ for $5 \mathrm{~min}$, followed by the addition of binding buffer (20 mM HEPES pH 7.9, 1 mM DTT, 0.1 $\mathrm{mM}$ EDTA, $50 \mathrm{mM} \mathrm{KCl}, 5 \%$ glycerol and $200 \mu \mathrm{g} / \mathrm{ml} \mathrm{BSA})$ and biotinylated oligo $(10 \mathrm{ng} / \mu \mathrm{l})$. To control for specificity of binding, for selected samples, a 5-fold excess of nonlabeled oligo was added prior to the addition of the biotinylated probe. Binding reaction mixtures were incubated for $30 \mathrm{~min}$ at room temperature. Protein-DNA complexes were separated on 5\% nondenaturing polyacrylamide gels in Tris-borate/EDTA buffer $(0.1 \mathrm{M}$ Tris, $0.09 \mathrm{M}$ boric acid containing $1 \mathrm{mM}$ EDTA) at $4^{\circ} \mathrm{C}$. After electrophoresis, gels were transferred to nylon membranes. Transferred oligos were immobilized by UV crosslinking for $3 \mathrm{~min}$. For detection of bound oligos, membranes were blocked using blocking buffer (Panomics EMSA Gel-Shift Kit) followed by the addition of Streptavidin-HRPO and blots were developed by ECL according to the manufacturer's instructions (Amersham, Arlington Heights, IL, U.S.A.). Commercially available biotinylated oligonucleotide encoding the Sp-1 motif 5'ATTCGATCGGGGCGGGGCGAG-3' was used as a canonical probe (Panomics, AY-1043).

\section{Combined EMSA and Western blotting (WEMSA)}

For this assay we used custom synthesized non-labeled, oligonucleotides encoding the Sp-1-like binding sequences located within the proximal promoter of CD14 (Fig. 1). The Sp-1-like oligos were as follows: (1) 5'GGGGGGTTGG-3' at position -345 to -336 , (2) 5'GTCCCTCCCCCT-3' at position -159 to -148 , (3) 5'AGGGGGCTGGC-3' at position -113 to -100 and (4) 5'AGAGGTGGGGAGG-3' at position -91 to -79 . WEMSA was performed essentially as described above, with the exception that separated DNA-protein complexes were transferred to nitrocellulose membrane instead of nylon membrane and probed with anti-Sp-1 for Western blotting. Blots were developed by ECL.

\section{RESULTS AND DISCUSSION}

\section{The Screening for Calcitriol-Regulated Binding of Sp-1 to DNA Response Elements in Human CD14 by WEMSA}

In many gene promoters it is possible to find multiple candidate binding sites for any particular transcription factor. Prior analysis of the human CD14 promoter indicated five CRE-like sites (6) and four candidate Sp-1 sites (sequences 1-4 in Fig. 1). These four sites show 70-85\% homology with canonical Sp-1-binding sequences. Using conventional EMSA, four labeled-oligonucleotides 
$-469$

AGTTAAATATCTGAGGATATTCAGGGACTTGGATTTGGTGGCAGGAGATCAA

CATAAACCAAGACAAGGAAGAAGTCAAAGAAATGAATCAAGTAGATTCTCT

GGGATATAAGGTAGGGGGATT GGGGGGTTGGATAGTGCAGAGTATGGTATG $-345 \quad$ Sp-1 (1) -336

GCCTAAGGCACTGAGGATCATCCTTTTCCCACACCCACCAGAGAAGGCTTAG

GCTCCCGAGTCAACAGGGCATTCACGCCTGGGGCGCCTGAGTCATCAGGACA

CTGCCAGGAGACACAGAACCCTAGATGCCCTGCAGAATCCTTCCTGTTACG $\underline{\mathbf{G}}$

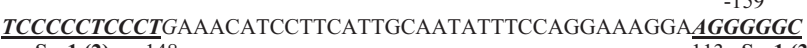
Sp-1 (2) -148 -113 Sp-1 (3)

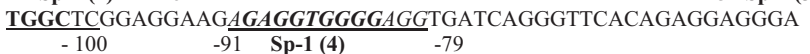
ACTGAATGACATCCCAGGATTACATAAACTGTCAGAGGCAGCCGAAGAGTT

CACA -1

FIg. 1: CD14 proxImal promoter. This region spanning 469 bp upstream of the transcriptional start site has been shown to be critical for the induction of CD14 transcription in response to calcitriol. Putative Sp-1like transcription factor-binding sites in this region were identified using the TFSEARCH program (http://www.cbrc.jp/research/db/TFSEARCH.html) and are highlighted in boldface, underlined and labeled as (1) 5'-

GGGGGGTTGG-3' at position -345 to -336, (2) 5'-GTCCCTCCCCCT-3' at position -159 to -148 , (3) 5'-AGGGGGCTGGC-3' at position -113 to -100 and (4) 5'-AGAGGTGGGGAGG-3' at position -91 to -79.

based on each of these putative sites would have been required to examine which, if any, were involved in Sp-1 binding. On the other hand, WEMSA does not require labeling of oligonucleotides as identification of the TF involved is based on Western blotting using specific antibodies. To evaluate further the effectiveness of WEMSA in screening for candidate DNA binding sites of a

$$
\begin{aligned}
& \text { A. Sp-1-like oligos } \\
& \text { 1. 5'-GGGGGGTTGG } \\
& \text { 2. 5'-TCCCCCTCCCT } \\
& \text { 3. 5'-GGGGGCTGGC } \\
& \text { 4. 5'-AGAGGTGGGGAGG }
\end{aligned}
$$

B. WEMSA

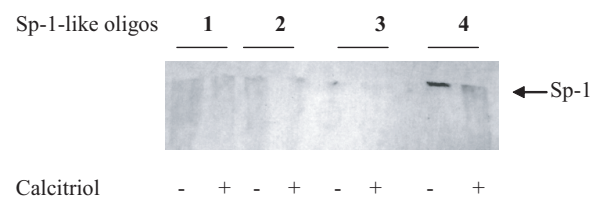

FIg. 2: WEMSA shows that Sp-1 binding to a speclfic sIte In the CD14 promoter is regulated by calcitriol. Serum starved THP-1 cells were either treated or not with $100 \mathrm{nM}$ calcitriol for $30 \mathrm{~min}$ followed by preparation of nuclear extracts for WEMSA as described in Materials and Methods. Nuclear extracts were incubated with four unlabelled oligos denoting putative Sp-1-like transcription factor-binding sites in CD14 promoter (A) and DNA protein complexes were separated by non-denaturing gel electrophoresis followed by transfer to nitrocellulose for Western blotting for Sp-1 (B). The results shown are from one of three independent experiments that yielded similar results.
A. WEMSA (Sp-1 canonical oligo)

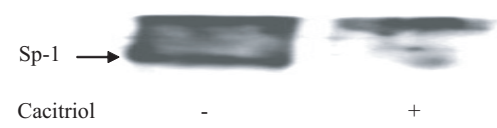

B. WEMSA (Sp-1-like oligo)

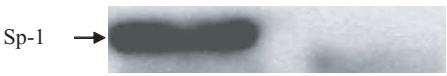

Cacitriol

Fig. 3: Sp-1 displays similar bindIng activity for both canonical and candidate CD14 promoter Sp-1 DNA binding sequences. Nuclear extract from calcitriol-treated and untreated THP-1 cells were used for WEMSA analysis using non-labeled canonical $(A)$ and $(B)$ the candidate CD14 promoter Sp-1 like oligo (sequence \#4 from Fig. 1).

transcription factor of interest, oligonucleotides were synthesized based on the four Sp-1-like sequences in the human CD14 promoter. THP-1 cells were treated with calcitriol and nuclear extracts were prepared and incubated with these four Sp-1-like sequences independently. DNA-protein complexes were then separated by $5 \%$ polyacrylamide gel electrophoresis, and separated DNA-protein complexes were transferred to nitrocellulose membranes for immunodetection of Sp-1. The results shown in Fig. 2 indicate that $\mathrm{Sp}-1$ bound to only one of the four candidate Sp-1 sites. This Sp-1 binding site (5'-AGAGGTGGGGAGG-3') located at positions -91 to -79 within the CD14 promoter has $85 \%$ identity to the canonical Sp-1 sequence (5'ATTCGATCGGGGCGGGGCGAG-3'). Sp-1 binding to this site was apparent in nuclear extracts from cells in the basal state and binding activity was negatively regulated by calcitriol. Whereas conventional Western blotting of nuclear extracts detected the presence of $\mathrm{Sp}-1$ in protein DNA complexes (Fig. 2), free Sp-1 not bound to DNA was not detected in the WEMSA assay (data was not shown). This was as expected since the $5 \%$ polyacrylamide gel would be unlikely to retain proteins of relatively smaller molecular mass such as uncomplexed free Sp-1. As an additional test for calcitriol-regulated Sp-1 binding activity, we conducted a parallel WEMSA analysis, in which the candidate Sp-1-like oligo from CD14 and a canonical Sp-1 oligo were used in parallel. Sp-1 binding to both the candidate and canonical sequences displayed similar activities in control and calcitriol-treated THP-1 


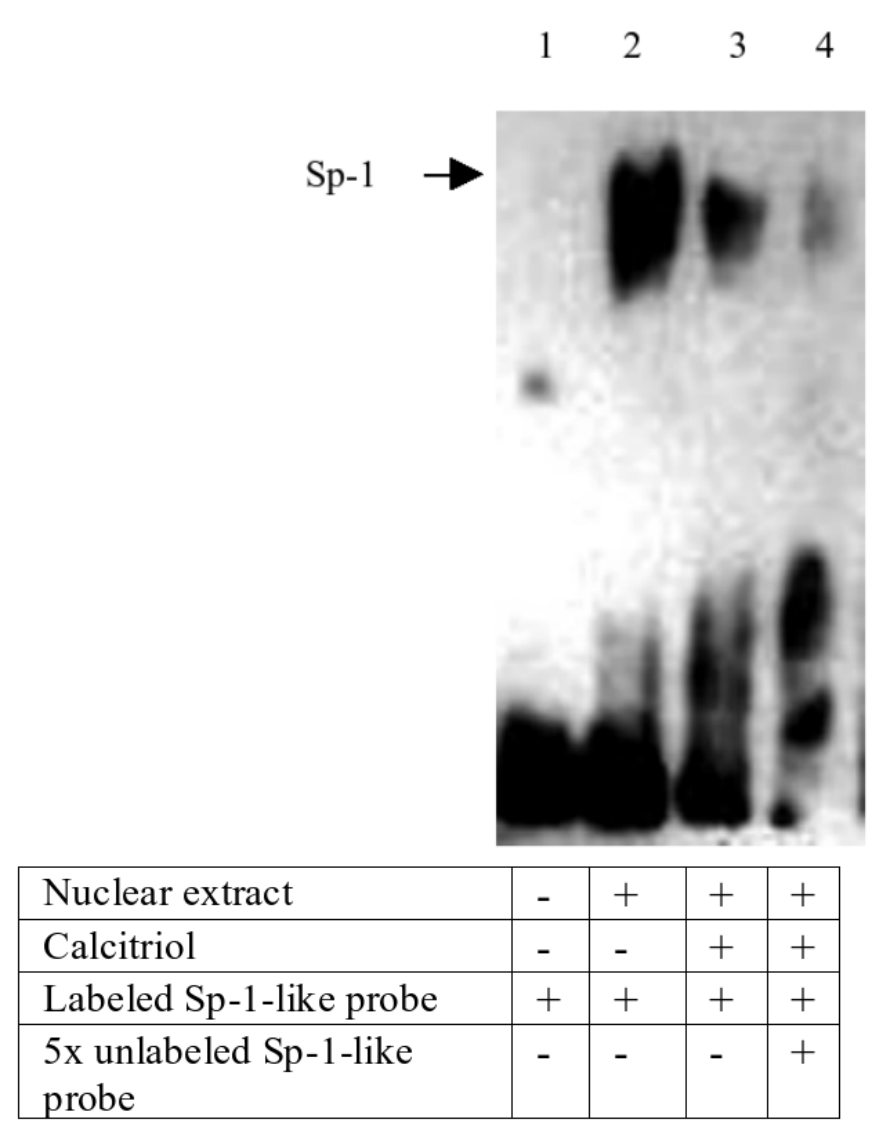

FIg. 4: EMSA analysis confirms results obtalned through WEMSA. EMSA using labeled Sp-1-like oligo spanning positions -91 to -79 of the CD14 promoter. Serum starved THP-1 cells were either untreated or treated with $100 \mathrm{nM}$ calcitriol for $30 \mathrm{~min}$ followed by preparation of nuclear extracts for EMSA as described in Materials and Methods. Lane 1, free labeled oligo. Lane 2, nuclear extract of untreated cells combined with Sp-1-like oligo. Lane 3, nuclear extract of calcitriol-treated cells combined with Sp-1-like oligo. Lane 4 represents nuclear extract from untreated cells combined with Sp-1-like oligo and unlabelled excess of Sp-1-like oligo.

cells (Figs. 3A and B).

Validation of WEMSA Analysis of Calcitriol-Regulated Sp-1 Binding to a Site in the CD14 Promoter by Classical EMSA

The analysis of calcitriol-regulated Sp-1 binding to this site in the CD14 promoter using WEMSA suggested that calcitriol negatively regulated Sp-1 binding to a specific DNA sequence (Figs. 2 and 3). In order to validate the results of WEMSA, we used the same candidate Sp-1-like oligo (5'-AGAGGTGGGGAGG-3') in a classical EMSA. THP-1 cells were treated with or without calcitriol and nuclear extracts were prepared and incubated with this Sp-1-like sequence. DNA-protein complexes were then separated by polyacrylamide gel electropheresis for EMSA. The results shown in Fig. 4 clearly demonstrate clacitriol-mediated down-regulation of Sp-1 binding to the Moeenrezakhanlou et al. - Identification of a Calcitriol-Regulated Sp-1 Site www.biologicalprocedures.com candidate Sp-1 binding sequence in the CD14 promoter, thereby corroborating the results obtained by WEMSA. Taken together, these results confirm that the candidate Sp-1-like binding site located at position -91 to -79 is a bona fide site for $\mathrm{Sp}-1$ binding and that calcitriol reduces Sp-1 binding to this position in the CD14 promoter.

The broad applicability of WEMSA is demonstrated by its use in identifying the involvement of $\mathrm{Sp}-1$, and in an earlier study, the transcription factor CREB, in calcitriolmediated CD14 expression (6). This demonstrates that WEMSA provides a suitable substitute for classical EMSA as a method to directly detect the potential involvement of transcription factors and their cognate DNA binding elements in gene regulation. In addition, WEMSA offers several advantages over classical EMSA which make this technique an attractive alternative to electrophoretic mobility shift assays. WEMSA is highly cost effective as compared to EMSA-based supershifts, as WEMSA does not require the use of modified oligonucleotides and antibodies can be used and recycled.

It is difficult to calculate the precise cost savings afforded by WEMSA over EMSA. However, our best estimate would be around $30 \%$. This is based on the cost of biotinylated oligonucleotides in EMSA as compared to unlabelled oligonucleotides in WEMSA and recycling of primary antibody in WEMSA as compared to EMSA. Moreover, antibodies suitable for conducting supershift assays in EMSA are often limited, as these are required to recognize epitopes under native conditions. In WEMSA, however, any antibody that is suitable for conventional Western blotting can be used. Thus, WEMSA provides an attractive option to classical EMSA as a method for the identification of transcription factors and their cognate DNA binding elements involved in regulating gene expression.

\section{ACKNOWLEDGMENTS}

This work was supported by Canadian Institutes of Health Research (CIHR) grants MOP-8633 \& MOP-83063 (NER) and FRN-38005 (DN). We thank Emily Thi (University of British Columbia, BC Canada) for reviewing the manuscript. 


\section{REFERENCES}

1. Yu X, Lin J, Zack DJ, and Qian J. Computational analysis of tissue-specific combinatorial gene regulation: predicting interaction between transcription factors in human tissues. Nucleic Acids Res 2006;34:4925-4936.

2. Messina DN, Glasscock J, Gish W, and Lovett M. Expression and the Construction of a Microarray to Interrogate Their An ORFeome-based Analysis of Human Transcription Factor Genes. Genome Res 2004;14:2041-2047.

3. Matys V, Fricke E, Geffers R, Gößling E, Haubrock M, Hehl R, Hornischer K, Karas D, Kel AE, Kel-Margoulis OV, Kloos DU, Land S, Lewicki-Potapov B, Michael H, Münch R, Reuter I, Rotert S, Saxel H, Scheer M, Thiele $S$, and Wingender E. TRANSFAC1: transcriptional regulation, from patterns to profiles. Nucleic Acids Res 2003;31:374-378.

4. Yan Y, Dalmasso G, Sitaraman S, and Merlin D. Characterization of the Human Intestinal CD98 Promoter and its Regulation by Interferon Gamma. Am J Physiol Gastrointest Liver Physiol 2007;292:G535G545.

5. Hmama Z, Nanda D, Sly L, Knutson KL, Herrera-Velit $\mathrm{P}$ and Reiner N. 1,25 dihydroxyvitamin $\mathrm{D}_{3}$ induced myeloid cell differentiation is regulated by a vitamin $\mathrm{d}$ receptor phosphatidylinositol 3 kinase signaling complex. J Exp Med 1999;190(11):1583-1594.

6. Moeenrezakhanlou A, Nandan D, Shephard L, and Reiner NE. 1 $\alpha, 25$-Dihydroxycholecalciferol activates binding of CREB to a CRE site in the CD14 promoter and drives promoter activity in a phosphatidylinositol-3 kinase-dependent manner. $J$ Leukoc Biol 2007;81:1311-1321.

7. Zhang DE, Hetherington JC, Gonzalez AD, Chen HM and Tenen GD. Regulation of CD14 Expression During Monocytic Differentiation Induced with 1, 25Dihydroxyvitamin $\mathrm{D}_{3}$. J Immunol 1994;153:3276.

8. Black RA, Black DJ and Azizkan-Clifford J. Sp1 and Kruppel-like Factor Family of Transcription Factors in Cell Growth Regulation and Cancer. J Cell Physiol 2001;188:143-160.

9. Giraudo E, Primo L, Audero E, Gerber HP, Koolwijk P, Soker S, Klagsbrun M, Ferrara $\mathrm{N}$ and Bussolino F. Tumor necrosis factor- $\beta$ regulates expression of vascular endothelial growth factor receptor- 2 and of its co-receptor neuropilin-1 in human vascular endothelial cells. J Biol Chem 1998;273:22128-22135.

10. Colmone A, Li Sh, and Wang Ch. Activating Transcription Factor/cAMP Response Element Binding Protein Family Member Regulated Transcription of CD1A. J Immunol 2006;177:7024-7032.

\section{PROTOCOLS}

Western Blotting Electrophoresis Mobility Shift Assay (WEMSA)

Reagents:

- Unlabelled and biotinylated Sp-1-like oligos for WEMSA and EMSA were from Invitrogen (Carlsbad, CA). Unlabelled and biotinylated canonical Sp-1 oligos and EMSA kit (EMSA Gel-Shift Kit \# AY 1288 P) were purchased from Panomics (Fremont, CA). Anti-Sp-1 antibody was from Upstate Cell Signaling Solutions (Lake Placid, NY)

- The human promonocytic cell line THP-1 was from American Type Culture Collection, Rockville, MD

- Cell culture medium: RPMI 1640 medium supplemented with 10\% (v/v) heat-inactivated fetal bovine serum (FBS), penicillin (100 units/ml) and streptomycin $(100 \mu \mathrm{g} / \mathrm{ml})$.

- 1 $\alpha$, 25-dihydroxyvitamin $\mathrm{D}_{3}$ (calcitriol) was obtained from Calbiochem (San Diego, CA).

Methods:

Nuclear extract:

- Nuclear fractions were prepared based on a protocol from the Skirball Institute of Biomolecular Medicine, NYU Medical Center with minor modifications (6).

Non-denaturing PAGE:

- Prepare 5\% non-denaturing polyacrylamide gels in Tris-borate/EDTA buffer $(0.1 \mathrm{M}$ Tris, $0.09 \mathrm{M}$ boric acid containing $1 \mathrm{mM}$ EDTA) and store at $4^{\circ} \mathrm{C}$.

- Mix 5-10 $\mu$ g of nuclear extracts from treated and control cells with relevant oligos and poly $(\mathrm{dI}-\mathrm{dC})(1 \mu \mathrm{g} / \mu \mathrm{l}) \mathrm{in}$ 
binding buffer (20 mM HEPES pH 7.9, $1 \mathrm{mM}$ DTT, $0.1 \mathrm{mM}$ EDTA, $50 \mathrm{mM} \mathrm{KCl}, 5 \%$ glycerol and $200 \mu \mathrm{g} / \mathrm{ml}$ BSA).

- Add loading buffer to the above mixture to fractionate samples on gel at $4^{\circ} \mathrm{C}$ for $2-3 \mathrm{hrs}$ using $100 \mathrm{~V}$.

Western blotting:

- Transfer PAGE separated proteins onto nitrocellulose membrane using Western blotting semi-dry transfer apparatus.

- Block the membrane and probe with anti-Sp-1-antibody as per the manufacturer's instructions (Upstate Cell Signaling Solutions).

- Develop with ECL system. 